\title{
On the use of total reflection x-ray topography for the observation of misfit dislocation strain at the surface of a $\mathrm{Si} / \mathrm{Ge}-\mathrm{Si}$ heterostructure
}

\author{
Patrick J. McNallya) \\ Research Institute for Networks and Communications Engineering (RINCE), Dublin City University, \\ Dublin 9, Ireland
}

G. Dilliway, J. M. Bonar, and A. Willoughby

Engineering Materials Department, University of Southampton, Highfield, Southampton, SO17 1BJ United Kingdom

T. Tuomi and R. Rantamäki

Optoelectronics Laboratory, Helsinki University of Technology, PO Box 3000, 02015 TKK, Finland

\author{
A. N. Danilewsky \\ D-79108, Freiburg, Germany \\ D. Lowney \\ Research Institute for Networks and Communications Engineering (RINCE), Dublin City University, \\ Dublin 9, Ireland
}

(Received 2 May 2000; accepted for publication 19 July 2000)

\begin{abstract}
Synchrotron x-ray topography was used in total reflection topography (TRT) mode to observe strain-induced surface bumps due to the presence of underlying misfit dislocations in strained-layer $\mathrm{SiGe}$ on $\mathrm{Si}$ epitaxial heterostructures. In these experiments, the $\mathrm{x}$ rays approached the sample surfaces at grazing incident angles below the critical angles for total external reflection for a number of reflections, and hence, surface strain features nominally less than a few tens of angstroms from the sample surface have been observed. These are similar to the surface bumpiness observed by atomic force microscopy, albeit on a much larger lateral length scale. The fact that TRT mode images were taken was confirmed by the observation of conventional backreflection topographic images of misfit dislocations in all samples when the grazing incidence angle became greater than the critical angle. (C) 2000 American Institute of Physics. [S0003-6951(00)04936-6]
\end{abstract}

There has been a growing interest over the past decade or so in the use of grazing incidence diffraction (GID) $\mathrm{x}$-ray topographic techniques for the analysis of thin surface crystal layers. ${ }^{1-6}$ For materials such as silicon, grazing incidence angles of the order of $0.1^{\circ}-1^{\circ}$ are typically used (angles which tend to be greater than the critical angle, $\alpha_{c}$ for the reflecting planes under analysis), which normally yield information about strain, defect, or dislocation distributions at penetration depths of the order of micrometers. ${ }^{5,6}$ However, in many modern epitaxial systems, very thin mismatched layers are grown close to the surface of the substrate, and the overall thickness of such active layers, which themselves may be heavily dislocated or strained, may be much smaller than the smallest achievable penetration depth $\left(t_{p}\right)$ using GID topography. One such system is that of the heteroepitaxy of thin $\mathrm{Si}_{1-x} \mathrm{Ge}_{x}$ layers on mismatched $\mathrm{Si}$ substrates.

In the past, a number of authors have used variants of the GID topography scheme, wherein grazing incident angles below the appropriate critical angles are used to image structures at or near the surface with $\mathrm{x}$-ray penetration depths as low as $140 \mathrm{~nm}^{7-9}$ With this type of problem in mind, the authors have investigated the possibility of using white beam synchrotron radiation total reflection $\mathrm{x}$-ray topography (TRT) to ascertain whether information about the structure

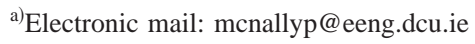

of epitaxially grown layers can be acquired at depths not exceeding $0.3 \mu \mathrm{m}$. The material examined consisted of three samples: (a) Sample "720" - Si substrate upon which was grown $\sim 1.7 \mu \mathrm{m}$ of $\mathrm{Si}_{1-x} \mathrm{Ge}_{x}$ virtual substrate with a $\mathrm{Ge}$ concentration varying linearly from $13.5 \%$ to $42 \%$ over the layer thickness followed by $\sim 0.3-\mu$ m-thick $\mathrm{Si}_{0.58} \mathrm{Ge}_{0.42}$ capping layer (growth temperature $=800^{\circ} \mathrm{C}$ ); (b) Sample " 775 " - Si substrate upon which was grown $\sim 1.36 \mu \mathrm{m}$ of $\mathrm{Si}_{1-x} \mathrm{Ge}_{x}$ virtual substrate with a $\mathrm{Ge}$ concentration varying linearly again from $12 \%$ to $42 \%$ followed by $\sim 0.3-\mu \mathrm{m}$-thick $\mathrm{Si}_{0.58} \mathrm{Ge}_{0.42}$ capping layer (growth temperature $=750^{\circ} \mathrm{C}$ ); and (c) Sample "774" - Si substrate upon which was grown $\sim 1.36 \mu \mathrm{m}$ of $\mathrm{Si}_{1-x} \mathrm{Ge}_{x}$ virtual substrate with a Ge concentration varying from $12 \%$ to $42 \%$ in five steps of $6 \%$, followed again by $\sim 0.3$ - $\mu$ m-thick $\mathrm{Si}_{0.58} \mathrm{Ge}_{0.42}$ active layer (growth temperature $=750^{\circ} \mathrm{C}$ ). In each case the total wafer thickness is $\sim 535 \mu \mathrm{m}$. Samples were grown by low pressure chemical vapor deposition in the [001] direction. The virtual substrates are relaxed from misfit strain and they seem to have relaxed through different relaxation mechanisms (especially for the linear-graded structures compared to the stepgraded one). As a result, a well-defined crosshatch pattern, with ridges running along two perpendicular $\langle 011\rangle$ directions, can be seen on the surface of the (001) plane.

The kinematical penetration depth $t_{p}$ (measured perpendicular to the surface $)^{1,6}$ at which the intensity of the outgo- 


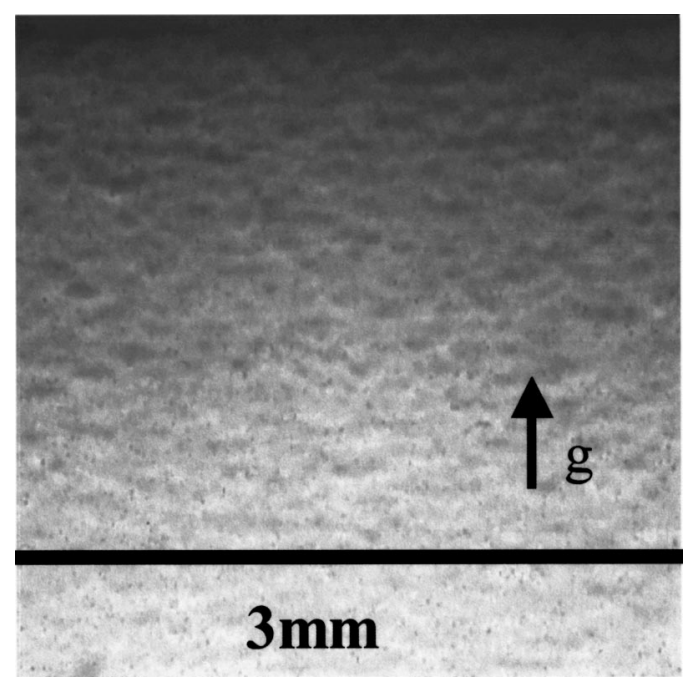

FIG. 1. 115 total reflection topograph of sample 775. Grazing incidence angle $\phi_{i}=0.05^{\circ}$. Nominal penetration depth $t_{p}=1.29 \AA$.

ing reflected beam has dropped to $1 / e$ times that of the incident beam due to absorption is satisfactory for most cases, but fails if $\phi_{i}, \phi_{h}<\alpha_{c}$, where $\phi_{i}\left(\phi_{h}\right)$ is the incidence (exit) angle. The dynamical penetration depth $t_{p}$ can be calculated from Ref. 10. Radiation only penetrates a few nanometers into the sample when $\phi_{i}<\alpha_{c}$.

The measurements were performed at HASYLAB am DESY, Hamburg, Germany, utilizing the continuous spectrum of synchrotron radiation from the DORIS storage ring bending magnet. The ring operated at a positron energy of $4.45 \mathrm{GeV}$ and at typical currents of 80-150 mA. The aforementioned Laue pattern of topographs was recorded on Kodak SO-343 and SO-181 high-resolution professional $\mathrm{X}$-ray films having an emulsion grain size of about $0.05 \mu \mathrm{m}$.
The distance from the sample to the film was $70 \mathrm{~mm}$. The angle of incidence varied between $\phi_{i}=0.05^{\circ}$ (nominal total external reflection) to $\phi_{i}=10^{\circ}$ (deeper penetration depths). The goniometer was moved by computer controlled stepper motors, which have step sizes of $0.1 \mu \mathrm{m}$ for $x-, y$-, and $z$-axis motions and $0.01^{\circ}$ steps for the angular tilt. Since a white beam is used in these experiments, the relative intensities of the harmonics in each topograph can be calculated. ${ }^{1,5,11,12}$

Three sets of figures, which were taken in the grazing incidence diffraction mode, are shown later. Figures 1, 3, and 4 were taken at grazing incidence angles of $\phi_{i}=0.05^{\circ}, \phi_{i}$ $=1.0^{\circ}$, and $\phi_{i}=10^{\circ}$, respectively, for sample 775 . Figure 1 is 115 reflection topograph, wherein the grazing angle is less than the critical angle for this wavelength $\left(\phi_{i}<\alpha_{c}\right)$ so in this case the dynamical equation ${ }^{10}$ was used to calculate the penetration depth. As expected it yielded a nominally small depth $(1.29 \AA)$, which confirms that these images are in a nominal total external reflection mode. In fact there are no clear images of misfit dislocations (MDs) to be seen. However, the image possesses a "bumpy" structure, which is presumably related to the underlying MDs. It has been observed that, for some strained epitaxial systems, the surface of the layer develops roughness or waviness, which correlated spatially with the positions of the underlying MDs, which partially relax the elastic mismatch strain. ${ }^{13-15}$ In the TRT image shown in Fig. 1 it is most likely that the strain fields due to this type of waviness are now being imaged. Figure 2 displays the corresponding three-dimensional (3D) atomic force microscopy (AFM) image for the sample. The strain induced surface waviness is easily observed in each image, with the surface bumps running along the direction of the $\langle 011\rangle$ family of misfit dislocations, as confirmed by Nomarski differential interference contrast microscopy and

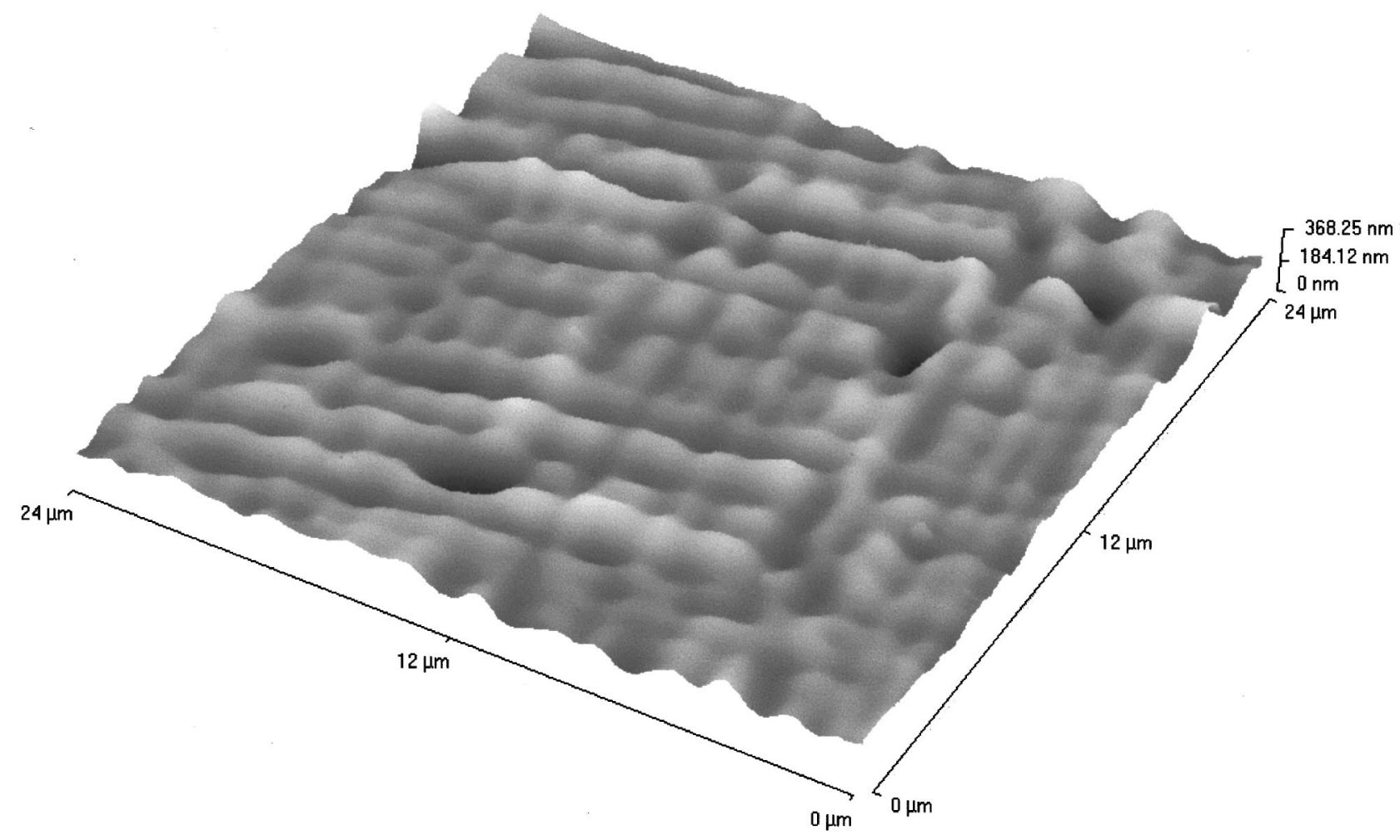

FIG. 2. 3D AFM image of sample 775 for a scan rate of $100 \mu \mathrm{m} / \mathrm{s}$. 


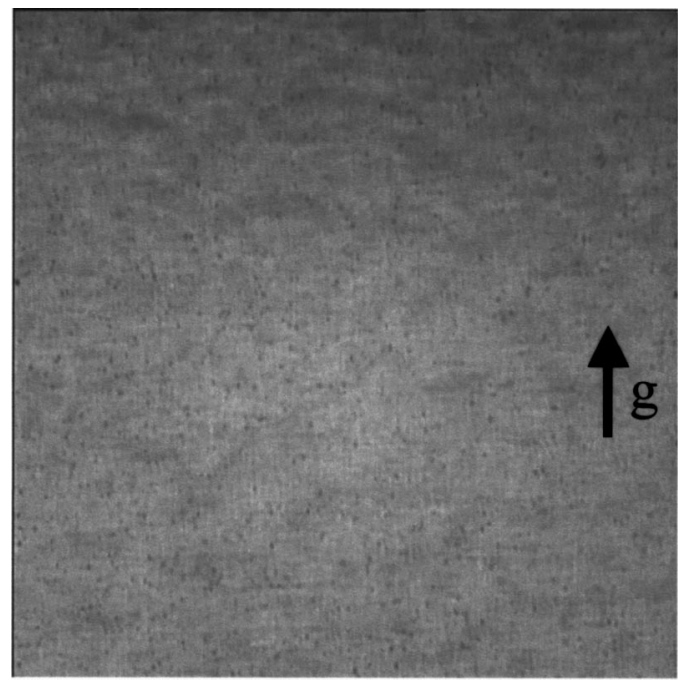

FIG. 3. 115 grazing incidence diffraction topograph of sample 775. Grazing incidence angle $\phi_{i}=1.0^{\circ}$. Nominal penetration depth $t_{p}=0.27 \mu \mathrm{m}$. Scale as for Fig. 1.

transmission electron microscopy. One can observe that the $\mathrm{x}$-ray topographic images of these wavy structures disappear as the penetration depths get larger (see Figs. 3-4). Figure 3 is a 115 reflection with $\phi_{i}=1.0^{\circ}$ and $t_{p}=0.27 \mu \mathrm{m}$. The crosshatched array of MDs is clearly seen, running parallel to

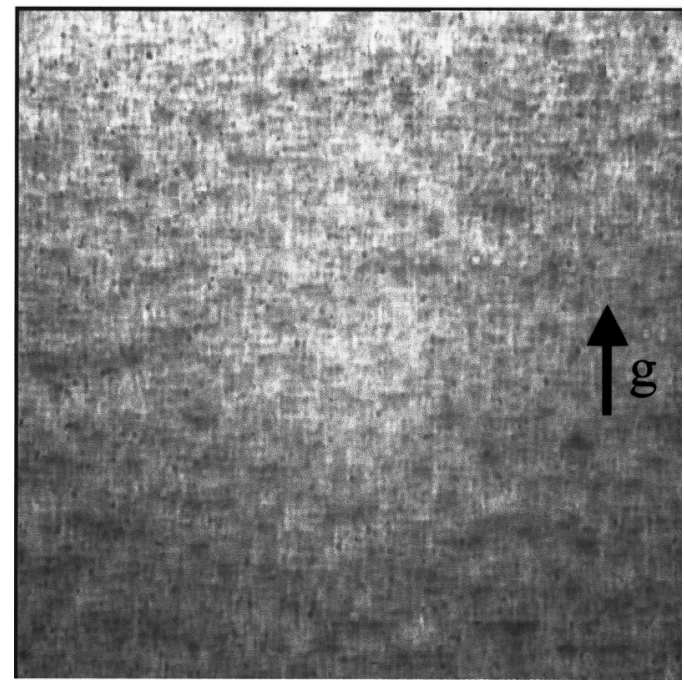

FIG. 4. 117 grazing incidence diffraction topograph of sample 775. Grazing incidence angle $\phi_{i}=10^{\circ}$. Nominal penetration depth $t_{p}=21.6 \mu \mathrm{m}$. Scale as for Fig. 1. the $\langle 011\rangle$ directions, and the surface structure has almost completely disappeared. The MD arrays are even more prominent in Fig. 4, a 117 reflection with $\phi_{i}=10^{\circ}$ and $t_{p}$ $=21.6 \mu \mathrm{m}$. At these penetration depths the signal from the wavy surface features, though still present, does not make up the largest contribution to the image, since a far greater underlying volume of sample is also diffracting. Similar observations are found for the other two samples.

To summarize, synchrotron x-ray topography has been used in TRT mode to observed strain induced surface waviness and bumps in strained layer SiGe on Si epitaxial structures, whose origin most likely is associated with the existence of misfit dislocations in the virtual substrate. Because the impinging $\mathrm{x}$ rays approach the sample surface at grazing angles below the critical angles for a number of reflections, surface strain features no more than nominally $1-10 \AA$ from the sample surface have been observed. These features displayed a remarkable resemblance to the surface bumpiness observed by AFM, albeit on a much larger lateral length scale.

The support of $\mathrm{T}$. Wroblewski at beamline $\mathrm{F}-1$, HASYLAB, Hamburg, Germany is greatly appreciated. This project was supported by the TMR-Contract No. ERBFMGECT950059 of the European Union and the Irish Forbairt International Collaboration Program.

${ }^{1}$ C. L. Kuo and J. C. Bilello, J. Appl. Phys. 62, 137 (1987).

${ }^{2}$ G. D. Gao, M. Dudley, and J. Wu, J. X-Ray Sci. Technol. 2, 195 (1990).

${ }^{3}$ G. D. Gao, S. W. Wilkins, A. W. Stephenson, and G. W. Pain, J. Appl. Phys. 60, 2604 (1992).

${ }^{4}$ D. V. Novikov, M. Ohler, R. Köhler, and G. Materlik, J. Phys. D: Appl. Phys. 28, A84 (1995)

${ }^{5}$ R. Rantamäki, T. Tuomi, P. J. McNally, J. Curley, and A. Danilewsky, J. X-Ray Sci. Technol. 8, 159 (1998).

${ }^{6}$ M. Dudley, J. Wu, and G. D. Yao, Nucl. Instrum. Methods Phys. Res. B 40/41, 388 (1989)

${ }^{7}$ A. M. Afansaev, P. A. Aleksandrov, R. M. Imamov, E. M. Pashaev, and V. Polovinkina, Phys. Status Solidi A 90, 419 (1985).

${ }^{8}$ T. Kitano, T. Ishikawa, J. Matsui, K. Akimoto, J. Mizuki, and Y. Kawase, Jpn. J. Appl. Phys., Part 2 26, L108 (1987).

${ }^{9}$ R. M. Imavov, A. A. Lomov, and D. V. Novikov, Phys. Status Solidi A 116, K133 (1989).

${ }^{10}$ H. Dosch, Phys. Rev. B 35, 2137 (1987)

${ }^{11}$ T. Tuomi, K. Naukkarinen, and P. Rabe, Phys. Status Solidi A 25, 93 (1974).

${ }^{12}$ M. Hart, J. Appl. Crystallogr. 8, 436 (1975).

${ }^{13}$ F. K. LeGoues, B. S. Meyerson, and J. F. Morar, Phys. Rev. Lett. 66, 2903 (1991)

${ }^{14}$ F. Jonsdottir, Mater. Res. Soc. Symp. Proc. 356, 45 (1995).

${ }^{15}$ E. A. Fitzgerald, D. G. Ast, P. D. Kirchner, G. D. Pettit, and J. M. Woodall, J. Appl. Phys. 63, 693 (1988) 